\title{
Welfare implications of mitigating investment uncertainty
}

\author{
Takayuki Ogawa ${ }^{1} \cdot J_{\text {Jun Sakamoto }}^{2}$ (1) \\ Received: 9 February 2020 / Accepted: 19 August 2021 / Published online: 15 September 2021 \\ (c) The Author(s) 2021
}

\begin{abstract}
This study explores the welfare implications of mitigating investment uncertainty in the context of Easley and O'Hara (Rev Financ Stud 22:1817-1843, 2009) While one may expect welfare gains by encouraging participation in financial markets by ambiguityaverse investors, we formally show that it hurts other investors and thus is not Paretoimproving without appropriate income transfers. We also examine the welfare effects of income redistribution among heterogeneous investors and government spending on investor education.
\end{abstract}

Keywords Ambiguity $\cdot$ Heterogeneous agents $\cdot$ Uncertainty $\cdot$ Welfare effects

JEL Classification D81 · G11 · G18

\section{Introduction}

Knight (1921) and Keynes (1937) emphasize the importance of distinguishing between risk and uncertainty (or ambiguity) when the probability law of the economy is unknown to some market participants. Decision making with ambiguity aversion is axiomatized by Gilboa and Schmeidler (1989) and Schmeidler (1989) and is applied

We are indebted to Yuichi Fukuta, Ichiro Gombi, Keiichi Hori, Shinsuke Ikeda, Yoshiyasu Ono, and the seminar participants at Osaka University for their helpful comments. This research is financially supported by JSPS KAKENHI Grant Numbers 19K01767 and 20K01669 for Ogawa and 19K23212 and 20K01669 for Sakamoto.

\footnotetext{
$凶 \quad$ Jun Sakamoto

jsakamoto@kobe-kiu.ac.jp

Takayuki Ogawa

tkogawa@osaka-ue.ac.jp

1 Faculty of Economics, Osaka University of Economics, 2-2-8 Osumi, Higashiyodogawa-ku, Osaka 533-8533, Japan

2 Faculty of Economics, Kobe International University, 9-1-6 Koyocho-naka, Higashinada-ku, Kobe, Hyogo 658-0032, Japan
} 
to various economic issues, prominently to the analysis of financial markets and asset prices. ${ }^{1}$ In this study, we investigate the welfare implications of reducing investment uncertainty, which, as per literature, is usually considered to be beneficial; additionally, we demonstrate that it hurts some investors and thus is not Pareto-improving without appropriate income transfers.

The theoretical model employed in this study is based on the work of Easley and O'Hara (2009), who develop a general equilibrium model with two types of investors: informed investors who precisely know the probability distribution of investment payoffs and uninformed investors who know this only imprecisely. ${ }^{2}$ After demonstrating that the uninformed investor chooses to participate in financial markets as the ambiguity about investment payoffs sufficiently dissipates, Easley and O'Hara mention that "regulation, particularly regulation of unlikely events, can moderate the effects of ambiguity, thereby increasing participation in financial markets and generating welfare gains" (p. 1818). Against their perspective, we formally show that it deteriorates the welfare of informed investors in their setting.

More accurately, we examine how individual welfare is affected by either increasing the minimum mean of investment payoffs perceived by the uninformed or decreasing the perceived maximum variance of investment payoffs. Both motivate the uninformed to demand holding more risky assets and push up the asset price, thereby making the informed reduce their risky asset holdings. We show that this corrects a distortion in the asset price but is undesirable for the informed, who has enjoyed the lower asset purchase price. In other words, the investor with more information loses a benefit arising from mispricing. The mitigation of investment uncertainty is thus harmful to the informed and consequently not desirable in a Pareto sense. The government needs to appropriately redistribute gains from the uninformed to the informed.

Several recent studies theoretically investigate the welfare effects attributed to various ambiguities. Most report a welfare improvement of decreasing ambiguity within a representative-agent framework. For instance, in a partial equilibrium setting in which an investor is ambiguous about a prediction model of stock returns, Chen et al. (2014) calibrate the welfare costs caused by the ambiguity. Ilut and Schneider (2014) estimate confidence shocks about future total factor productivity and find that they are a major source of business cycle frequency movements. ${ }^{3}$ Alonso and Prado (2015) document a large benefit of removing all consumption fluctuations when a consumer confronts ambiguity about the transition probability of stock's dividend growth rates. As an exception, Easley et al. (2014) demonstrate the welfare effect to be ambiguous when some traders face uncertainty about the trading strategies of others. They focus only on the welfare of the ambiguity-facing traders and do not discuss a spillover effect on the others. In contrast with the existing literature, we analyze all individuals' welfare

\footnotetext{
1 For theoretical applications, refer to e.g., Dow and da Costa Werlang (1992), Epstein and Wang (1994, 1995), Chen and Epstein (2002), Epstein and Miao (2003), Cao et al. (2005), and Easley and O'Hara (2009). See Anderson et al. (2009) and Antoniou et al. (2015) for empirical studies and Guidolin and Rinaldi (2013) for a survey.

2 In Easley and O'Hara (2009), the informed investor is called the sophisticate and the uninformed investor is called the naïf. However, we believe that our naming is more precise, at least in our context, since all investors rationally make decisions with given priors on the probability distribution.

3 See also (Cagetti et al. 2002; Bidder and Smith 2012) for the effect of ambiguity on business cycles.
} 
in a heterogeneous-agents model and determine how to achieve a Pareto improvement through fiscal policy.

The remainder of this study is organized as follows. Section 2 presents the structure of the model. Section 3 analyzes the welfare of each investor to show that a Pareto improvement cannot be attained solely by mitigating investment uncertainty. Section 4 considers two kinds of redistribution policies between the informed and the uninformed, finding that, as investment uncertainty eases, a lump-sum income transfer from the improved uninformed to the damaged informed can lead to a Pareto improvement, but an investment subsidy cannot. Section 5 extends the analysis by considering government spending on investor education and endogenously determining the degree of ambiguity perceived by the uninformed. Section 6 concludes the study and provides directions for future research.

\section{The model}

The proposed model is based on Easley and O'Hara (2009). A closed economy, which lasts two periods, is inhabited by two types of investors, $j=I, U$. There exist one safe asset with a zero rate of return and one risky asset whose stochastic payoff realized in the second period is normally distributed. ${ }^{4}$ Only a part of the investors (called informed investors, $I)$ know the true mean and variance of the stochastic payoff, $(\hat{v}, \hat{\sigma})$, whereas the remaining (called uninformed investors, $U$ ) face ambiguity regarding the payoff distribution and subjectively possess a possible set of mean payoff $\left\{\bar{v}_{\min }, \ldots, \bar{v}_{\max }\right\}$ and that of variance $\left\{\bar{\sigma}_{\min }, \ldots, \bar{\sigma}_{\max }\right\}$. It is assumed to satisfy

$$
0<\bar{v}_{\min }<\hat{v}<\bar{v}_{\max }, \quad 0<\bar{\sigma}_{\min }<\hat{\sigma}<\bar{\sigma}_{\max }
$$

At the beginning of the first period, each investor is endowed with a constant amount of the safe and risky assets, $\bar{m}(>0)$ and $\bar{x}(>0)$, and chooses a portfolio, $\left(m_{j}, x_{j}\right)$, where $m_{j}$ and $x_{j}$ denote the investor $j$ 's demand for the safe and risky assets, respectively. Given the current price of the risky asset, $p$, the budget constraint in the first period requires that

$$
\bar{m}+p \bar{x}=m_{j}+p x_{j}
$$

In the second period, investors consume their wealth entirely. The realized consumption, $\tilde{c}_{j}$, is stochastic and given by

$$
\tilde{c}_{j}=m_{j}+\tilde{v} x_{j}
$$

where $\tilde{v}$ represents the stochastic payoff when holding the risky asset.

\footnotetext{
${ }^{4}$ Easley and O'Hara (2009) model contains two risky assets, one of which has no uncertainty regarding payoffs. For the sake of simplicity, we do not introduce it. This is because, as long as the payoffs of two risky assets are independently distributed, the welfare effect of adding one more asset with no uncertainty is negligible. See Huang et al. (2017) for the case in which the payoffs of risky assets correlate.
} 
All investors share the same preference with respect to consumption $\tilde{c}_{j}$, in which the absolute risk aversion $\Gamma(>0)$ is constant. In this case, the utility maximization problem reduces to the mean-variance approach (see Appendix A). For the informed investor who knows the true probability distribution, it is

$$
\max _{x_{I}} U_{I}=(\bar{m}+p \bar{x})+(\hat{v}-p) x_{I}-\frac{\Gamma \hat{\sigma}}{2}\left(x_{I}\right)^{2} .
$$

The first term on the right-hand side represents the value of the initial endowment, the second term is the excess return of holding the risky asset, and the third term is the disutility from risk-taking. Solving this problem yields the informed's demand function for the risky asset:

$$
x_{I}=\frac{\hat{v}-p}{\Gamma \hat{\sigma}} .
$$

By using this demand function to eliminate $\hat{v}-p$ from (4), we can reduce the utility function to

$$
U_{I}=(\bar{m}+p \bar{x})+\frac{\Gamma \hat{\sigma}}{2}\left(x_{I}\right)^{2}
$$

While the payoff of the risky asset is not only more profitable but also more volatile than that of the safe asset, the former benefit necessarily dominates the latter disutility in the mean-variance approach. Welfare is thus increasing with respect to the risky asset holdings, $x_{I}(\geq 0)$.

As axiomatized by Gilboa and Schmeidler (1989), when those who are ambiguityaverse cannot place a unique prior on the probability distribution, they aim to maximize the minimum expected utility. In the present context, the uninformed investor chooses a portfolio expecting the worst scenario, which is that the minimum (maximum) mean payoff and the maximum variance are realized when taking a long (short) position in the risky asset. That is,

$$
\max _{x_{U}} \quad U_{U}= \begin{cases}(\bar{m}+p \bar{x})+\left(\bar{v}_{\min }-p\right) x_{U}-\frac{\Gamma \bar{\sigma}_{\max }}{2}\left(x_{U}\right)^{2} & \text { if } x_{U} \geq 0 \\ (\bar{m}+p \bar{x})+\left(\bar{v}_{\max }-p\right) x_{U}-\frac{\Gamma \bar{\sigma}_{\max }}{2}\left(x_{U}\right)^{2} & \text { if } x_{U} \leq 0\end{cases}
$$

Hence, the uninformed holds the risky asset, as shown in the equation below:

$$
x_{U}= \begin{cases}\frac{\bar{v}_{\min }-p}{\Gamma \bar{\sigma}_{\max }} & \text { if } p<\bar{v}_{\min } \\ 0 & \text { if } \bar{v}_{\min } \leq p \leq \bar{v}_{\max } \\ \frac{\bar{v}_{\max }-p}{\Gamma \bar{\sigma}_{\max }} & \text { if } \bar{v}_{\max }<p\end{cases}
$$

Welfare is given by

$$
U_{U}=(\bar{m}+p \bar{x})+\frac{\Gamma \bar{\sigma}_{\max }}{2}\left(x_{U}\right)^{2} .
$$


The uninformed investor evaluates benefits from holding the risky asset not by the true payoff variance $\hat{\sigma}$ but by the expected one $\bar{\sigma}_{\max }$.

The equilibrium condition in the risky asset market requires

$$
(1-\mu) x_{I}+\mu x_{U}=\bar{x}(>0)
$$

where $\mu(\in(0,1))$ denotes an exogenous ratio of the uninformed investors in the total population. Since the right-hand side of this equation is positive, the case where all investors take short positions cannot be equilibrium - that is, from (7), the asset price $p$ necessarily lies below $\bar{v}_{\max }$ in equilibrium. Hence, there are two cases depending on the uninformed's portfolio choice: ${ }^{5}$

Case 1 (Nonparticipating equilibrium): When the equilibrium asset price satisfies $\bar{v}_{\min } \leq p \leq \bar{v}_{\max }$, the uninformed investor does not demand the risky asset, namely, $x_{U}=0$. Following Easley and O'Hara (2009), we call this case the nonparticipating equilibrium.

Case 2 (Participating equilibrium): If the risky asset price is sufficiently low, such that $p<\bar{v}_{\text {min }}$, then all investors exhibit positive demand for the risky asset, namely, $x_{j}>0$. This case is called the participating equilibrium.

We now derive the equilibrium condition and individual welfare in each case. First, in Case 1, these come from (4) through (8):

$$
\begin{aligned}
& x_{U}^{N}=0, \quad x_{I}^{N}=\frac{\bar{x}}{1-\mu}, \quad p^{N}=\hat{v}-\frac{\Gamma \hat{\sigma} \bar{x}}{1-\mu}, \\
& U_{U}^{N}=\bar{m}+p^{N} \bar{x}, \quad U_{I}^{N}=\left(\bar{m}+p^{N} \bar{x}\right)+\frac{\Gamma \hat{\sigma}}{2}\left(x_{I}^{N}\right)^{2},
\end{aligned}
$$

where the superscript $N$ stands for a variable in the nonparticipating equilibrium. It is evident that the ambiguity parameters $\left(\bar{v}_{\min }, \bar{v}_{\max }, \bar{\sigma}_{\max }\right)$ have no effect on individuals' welfare as long as the uninformed investors do not demand the risky asset. The nonparticipating equilibrium exists if and only if the following condition is met:

\section{Condition 1 (The existence condition for the nonparticipating equilibrium)}

$$
\bar{v}_{\text {min }} \leq p^{N} \leq \bar{v}_{\text {max }}, \quad \text { or equivalently, } \quad \bar{v}_{\text {min }} \leq \hat{v}-\frac{\Gamma \hat{\sigma} \bar{x}}{1-\mu} \leq \bar{v}_{\text {max }}
$$

Next, we consider Case 2. Using (4) through (8) yields the participating equilibrium, indexed by the superscript $P$, as follows:

$$
\begin{aligned}
& x_{U}^{P}=\frac{1-\mu}{\Gamma\left[(1-\mu) \bar{\sigma}_{\max }+\mu \hat{\sigma}\right]}\left[\bar{v}_{\min }-\left(\hat{v}-\frac{\Gamma \hat{\sigma} \bar{x}}{1-\mu}\right)\right], \\
& x_{I}^{P}=\frac{\mu}{\Gamma\left[(1-\mu) \bar{\sigma}_{\max }+\mu \hat{\sigma}\right]}\left[\left(\hat{v}-\bar{v}_{\min }\right)+\frac{\Gamma \bar{\sigma}_{\max } \bar{x}}{\mu}\right]=x_{I}^{N}-\frac{\mu}{1-\mu} x_{U}^{P},
\end{aligned}
$$

\footnotetext{
5 The equilibrium condition for the safe asset is automatically satisfied because Walras' law holds.
} 


$$
\begin{aligned}
p^{P} & =\frac{(1-\mu) \bar{\sigma}_{\max } \hat{v}+\mu \hat{\sigma} \bar{v}_{\min }-\Gamma \hat{\sigma} \bar{\sigma}_{\max } \bar{x}}{(1-\mu) \bar{\sigma}_{\max }+\mu \hat{\sigma}}=p^{N}+\frac{\mu \Gamma \hat{\sigma}}{1-\mu} x_{U}^{P}, \\
U_{U}^{P} & =\left(\bar{m}+p^{P} \bar{x}\right)+\frac{\Gamma \bar{\sigma}_{\max }}{2}\left(x_{U}^{P}\right)^{2}, \\
U_{I}^{P} & =\left(\bar{m}+p^{P} \bar{x}\right)+\frac{\Gamma \hat{\sigma}}{2}\left(x_{I}^{P}\right)^{2},
\end{aligned}
$$

where $x_{I}^{N}$ and $p^{N}$ in the second and third equations are given in (9) and are independent of the ambiguity parameters. The participating equilibrium exists if and only if the following condition is met:

\section{Condition 2 (The existence condition for the participating equilibrium)}

$$
p^{P}<\bar{v}_{\text {min }}, \quad \text { or equivalently, } \quad \hat{v}-\frac{\Gamma \hat{\sigma} \bar{x}}{1-\mu}<\bar{v}_{\text {min }} .
$$

Clearly, $x_{N}^{P}$ and $x_{S}^{P}$ in (10) have positive values under Condition 2 and (1).

From the second and third equations in (10), we can establish the following lemma:

Lemma 1 The participation of the uninformed investors $\left(x_{U}^{P}>0\right)$ raises the risky asset price $\left(p^{P}>p^{N}\right)$, thereby depressing the risky asset demand of the informed investors $\left(x_{I}^{P}<x_{I}^{N}\right)$.

\section{Welfare}

In this section we analyze the welfare effects of reducing uncertainty in the risky asset market, interpreted as either increasing the minimum mean payoff $\bar{v}_{\text {min }}$ or decreasing the maximum variance $\bar{\sigma}_{\max }$, perceived by the uninformed investors.

Easley and O'Hara (2009) suggest how to mitigate investment uncertainty through policy interventions such as investor education and financial regulations. Education for the uninformed investors undeniably helps correct their wrong beliefs regarding the payoff distribution. Regulations, which are designed to make the worst case perceived by the uninformed impossible, also play the same role. For example, a government guarantee against risky investment raises the perceived minimum mean payoff, whereas the Investment Act, which forces mutual funds to diversify investment, decreases the perceived maximum variance. These interventions intend to improve welfare by inducing the uninformed to participate in the risky asset market. However, we demonstrate that they hurt the informed participants without appropriate income transfers. For this purpose, this section considers welfare effects of exogenous shifts in the perceived priors on the payoff distribution - an increase in the perceived minimum mean payoff from $\bar{v}_{\text {min }}$ to $\bar{v}_{\text {min }}^{\prime}\left(\in\left(\bar{v}_{\text {min }}, \hat{v}\right)\right)$ and a decrease in the perceived maximum variance from $\bar{\sigma}_{\max }$ to $\bar{\sigma}_{\max }^{\prime}\left(<\bar{\sigma}_{\max }\right)^{6}$

\footnotetext{
6 In Sect. 5, we extend the analysis by treating endogenous shift in these priors through government spending on investor education.
} 
Suppose first that the economy is initially in the nonparticipating equilibrium. Compare Conditions 1 and 2 to understand that an increase in the perceived minimum mean payoff $\bar{v}_{\text {min }}$ changes the equilibrium from Case 1 to Case $2 .^{7}$ This means that the mitigation of investment uncertainty motivates the uninformed investor to participate in the risky asset market. As already shown in Lemma 1, the resulting participation in the risky asset market by the uninformed investor raises the market price of the risky asset and then lowers the informed's demand for holding the risky asset. On one hand, the uninformed's welfare definitely improves, since both the value of endowment and the amount of risky asset holdings increase:

$$
U_{U}^{P}-U_{U}^{N}=\underbrace{\left(p^{P}-p^{N}\right) \bar{x}}_{(+)}+\underbrace{\frac{\Gamma \bar{\sigma}_{\max }}{2}\left(x_{U}^{P}\right)^{2}}_{(+)}>0
$$

which is obtained by subtracting the fourth equations in (9) from the fourth equation in (10). On the other hand, the informed confronts two conflicting effects-a rise in the value of endowment and a decrease in risky asset holdings:

$$
U_{I}^{P}-U_{I}^{N}=\underbrace{\left(p^{P}-p^{N}\right) \bar{x}}_{(+)}+\underbrace{\frac{\Gamma \hat{\sigma}}{2}\left[\left(x_{I}^{P}\right)^{2}-\left(x_{I}^{N}\right)^{2}\right]}_{(-)} .
$$

By substituting the equilibrium values given in (9) and (10), we demonstrate that the first beneficial effect is dominated by the second harmful effect,

$$
U_{I}^{P}-U_{I}^{N}=-\frac{\mu^{2} \hat{\sigma} x_{U}^{P}\left\{(1-\mu)\left[\left(\hat{v}-\bar{v}_{\min }^{\prime}\right)+2 \Gamma\left(\bar{\sigma}_{\max }-\hat{\sigma}\right) \bar{x}\right]+\Gamma \hat{\sigma} \bar{x}\right\}}{2(1-\mu)^{2}\left[(1-\mu) \bar{\sigma}_{\max }+\mu \hat{\sigma}\right]}<0,
$$

and whose sign is ensured by (1). Therefore, the mitigation in investment uncertainty, represented by an increase in $\bar{v}_{\text {min }}$, enhances the uninformed's welfare but worsens the informed's welfare.

To evaluate the aggregate effect, we define the social welfare weighted by the population size as

$$
U_{W} \equiv(1-\mu) U_{I}+\mu U_{U}
$$

Using (11) and (13) implies that an increase in $\bar{v}_{\text {min }}$ globally improves social welfare:

$$
U_{W}^{P}-U_{W}^{N}=\frac{(1-\mu) \mu}{2 \Gamma\left[(1-\mu) \bar{\sigma}_{\max }+\mu \hat{\sigma}\right]}\left[\bar{v}_{\min }^{\prime}-\left(\hat{v}-\frac{\Gamma \hat{\sigma} \bar{x}}{1-\mu}\right)\right]^{2}>0 .
$$

\footnotetext{
7 Note that the threshold between Case 1 and Case 2 is independent of $\bar{\sigma}_{\max }$. If the economy stays in the nonparticipating equilibrium after the shock, a change in $\bar{v}_{\min }$ as well as $\bar{\sigma}_{\max }$ has no effect on equilibrium variables, as seen in (9).
} 
See Appendix B for the derivations of (13) and (15).

Next, suppose that the economy is initially in the participating equilibrium, satisfying Condition 2. We can derive an effect within the participating equilibrium by totally differentiating (10) and (15) with respect to either $\bar{v}_{\min }$ or $\bar{\sigma}_{\text {max }}$ :

$$
\begin{aligned}
& \frac{\mathrm{d} U_{U}^{P}}{\mathrm{~d} \bar{v}_{\text {min }}}>0, \quad \frac{\mathrm{d} U_{I}^{P}}{\mathrm{~d} \bar{v}_{\text {min }}}<0, \quad \frac{\mathrm{d} U_{W}^{P}}{\mathrm{~d} \bar{v}_{\text {min }}}>0, \\
& \frac{\mathrm{d} U_{U}^{P}}{\mathrm{~d} \bar{\sigma}_{\max }}<0, \quad \frac{\mathrm{d} U_{I}^{P}}{\mathrm{~d} \bar{\sigma}_{\max }}>0, \quad \frac{\mathrm{d} U_{W}^{P}}{\mathrm{~d} \bar{\sigma}_{\max }}<0 .
\end{aligned}
$$

See Appendix B for the analytical details. The implication is the same as the previous argument about a regime shift- that is, as the asset demand of uninformed investors expands, the asset price rises and the asset holdings of informed investors decreases. The welfare effect is positive for the uninformed but negative for the informed.

The above results are summarized as follows:

Proposition 1 On the risky asset with uncertainty, both an increase in the minimum of possible mean payoff $\bar{v}_{\text {min }}$ and a decrease in the maximum of possible payoff variance $\bar{\sigma}_{\text {max }}$ raise the social welfare, as defined by (14). However, they do not lead to a Pareto improvement - that is, uninformed investors are better off, whereas informed investors are worse off.

Against the perspective of Easley and O'Hara (2009), with no further policy intervention, the mitigation in investment uncertainty is not desirable in a Pareto sense.

Thus far, we have focused on the effects of reducing uncertainty. One may be interested in a comparison with a shift in the true probability distribution. In Appendix C, we show that (i) an increase in the true mean payoff $\hat{v}$ achieves a Pareto improvement even without policy interventions and (ii) a decrease in the true payoff variance $\hat{\sigma}$ is Pareto-improving at least for a small $\mu$. An increase in $\hat{v}$ makes the risky asset more profitable, thereby raising the value of the initial endowment. This is beneficial to all individuals and occurs even if the economy lies in the nonparticipating equilibrium. ${ }^{8}$ Moreover, this beneficial effect definitely outweighs the harmful effect arising in the participating equilibrium - the raised asset price decreases the asset holdings of uninformed investors and deteriorates the uninformed's welfare. An increase in $\hat{v}$ is thus always desirable in a Pareto sense, while an increase in $\bar{v}_{\text {min }}$ is not (Proposition 1). A decrease in $\hat{\sigma}$ not only has a similar desirable effect but also reduces the excess return of holding the risky asset. Due to these conflicting effects, the welfare effect on the informed, who holds the risky asset more compared with the uninformed, is generically ambiguous, while the uninformed is better off. The implications of remedying distortions caused by uncertainty fairly differ from those of changes in the true payoff distribution and thus is worth being analyzed independently, as we have done.

\footnotetext{
8 Note that a change in $\bar{v}_{\min }$ has no effect when the economy is in the nonparticipating equilibrium.
} 


\section{Redistribution policy}

Proposition 1 states that mitigating investment uncertainty has a positive effect on the uninformed's welfare, which is larger than a negative effect on the informed's welfare in the absolute value, so that the social welfare improves. This section examines whether policymakers can achieve a Pareto improvement by implementing income transfers from the improved investors to the damaged investors simultaneously when investment uncertainty eases.

\subsection{Lump-sun transfers}

A lump-sum transfer from the uninformed investors to the informed investors is incorporated into the previous model. Letting $\tau_{j}^{t}$ be a lump-sum subsidy to individual $j(=I, U)$ in period $t(=1,2)$, we can rewrite the investor's budget constraints (2) and (3) as

$$
\bar{m}+p \bar{x}+\tau_{j}^{1}=m_{j}+p x_{j}, \quad \tilde{c}_{j}=m_{j}+\tilde{v} x_{j}+\tau_{j}^{2}
$$

A negative $\tau_{j}^{t}(<0)$ means that a lump-sum taxation is imposed. ${ }^{9}$ The balanced budget of the government requires

$$
(1-\mu) \tau_{I}^{t}+\mu \tau_{U}^{t}=0 \text { for } t=1,2
$$

In the presence of redistribution, individual utility is expressed by

$$
\hat{U}_{I} \equiv U_{I}+\left(\tau_{I}^{1}+\tau_{I}^{2}\right), \quad \hat{U}_{U} \equiv U_{U}-\frac{1-\mu}{\mu}\left(\tau_{I}^{1}+\tau_{I}^{2}\right)
$$

where $U_{I}$ and $U_{U}$ are given in (4) and (6), respectively (see Appendix A). Thus, the lump-sum transfer does not alter the demand functions for the risky asset (5) and (7), the equilibrium values $\left(x_{U}, x_{I}, p\right)$ in (9) and (10), and Conditions 1 and 2.

Suppose that the economy is at first in the nonparticipating equilibrium with null transfer and that the government implements the transfer as soon as an increase in $\bar{v}_{\min }$ shifts the equilibrium from Case 1 to Case 2. Consider a moderate transfer that compensates the welfare loss incurred by the informed investor. A Pareto improvement requires

$$
\begin{aligned}
& \hat{U}_{I}^{P}-\hat{U}_{I}^{N}=\left(U_{I}^{P}-U_{I}^{N}\right)+\left(\tau_{I}^{1}+\tau_{I}^{2}\right) \geq 0, \\
& \hat{U}_{U}^{P}-\hat{U}_{U}^{N}=\left(U_{U}^{P}-U_{U}^{N}\right)-\frac{1-\mu}{\mu}\left(\tau_{I}^{1}+\tau_{I}^{2}\right)>0,
\end{aligned}
$$

or equivalently,

\footnotetext{
9 Throughout this study, we assume that $\bar{m}$ is large enough to satisfy $\bar{m}+\tau_{j}^{1}+\tau_{j}^{2}>0$.
} 


$$
\underbrace{-\left(U_{I}^{P}-U_{I}^{N}\right)}_{(+)} \leq \tau_{I}^{1}+\tau_{I}^{2}<\frac{\mu}{1-\mu}\left(U_{U}^{P}-U_{U}^{N}\right),
$$

where $U_{I}^{P}-U_{I}^{N}<0$ is shown in (13). ${ }^{10}$

The lump-sum transfer does not affect the level of aggregate welfare (14) and thus the social benefit remains (15). Then, it holds that

$$
\begin{aligned}
\hat{U}_{W}^{P}-\hat{U}_{W}^{N} & \equiv(1-\mu)\left(\hat{U}_{I}^{P}-\hat{U}_{I}^{N}\right)+\mu\left(\hat{U}_{U}^{P}-\hat{U}_{U}^{N}\right) \\
& =(1-\mu)\left(U_{I}^{P}-U_{I}^{N}\right)+\mu\left(U_{U}^{P}-U_{U}^{N}\right), \\
& =U_{W}^{P}-U_{W}^{N}>0,
\end{aligned}
$$

which ensures

$$
-\left(U_{I}^{P}-U_{I}^{N}\right)<\frac{\mu}{1-\mu}\left(U_{U}^{P}-U_{U}^{N}\right)
$$

Therefore, the Pareto-improving transfer, which satisfies (17), exists whenever the social welfare is increased.

We can similarly find a Pareto-improving policy in the case wherein the economy initially lies in the participating equilibrium and stays within the participating equilibrium after the uncertainty is mitigated. The requirement of Pareto improvement is as follows:

$$
\begin{aligned}
& \underbrace{-\frac{\mathrm{d} U_{I}^{P}}{\mathrm{~d} \bar{v}_{\text {min }}}}_{(+)} \leq \frac{\mathrm{d}\left(\tau_{I}^{1}+\tau_{I}^{2}\right)}{\mathrm{d} \bar{v}_{\text {min }}}<\frac{\mu}{1-\mu} \frac{\mathrm{d} U_{U}^{P}}{\mathrm{~d} \bar{v}_{\text {min }}}, \\
& \underbrace{\frac{\mathrm{d} U_{I}^{P}}{\mathrm{~d} \bar{\sigma}_{\text {max }}}}_{(+)} \leq-\frac{\mathrm{d}\left(\tau_{I}^{1}+\tau_{I}^{2}\right)}{\mathrm{d} \bar{\sigma}_{\max }}<-\frac{\mu}{1-\mu} \frac{\mathrm{d} U_{U}^{P}}{\mathrm{~d} \bar{\sigma}_{\text {max }}},
\end{aligned}
$$

where $\mathrm{d} U_{I}^{P} / \mathrm{d} \bar{v}_{\min }<0$ and $\mathrm{d} U_{I}^{P} / \mathrm{d} \bar{\sigma}_{\max }>0$ are from (16). Since $\mathrm{d} U_{W}^{P} / \mathrm{d} \bar{v}_{\min }>0$ and $\mathrm{d} U_{W}^{P} / \mathrm{d} \bar{\sigma}_{\max }<0$ imply

$$
-\frac{\mathrm{d} U_{I}^{P}}{\mathrm{~d} \bar{v}_{\min }}<\frac{\mu}{1-\mu} \frac{\mathrm{d} U_{U}^{P}}{\mathrm{~d} \bar{v}_{\min }} \quad \text { and } \quad \frac{\mathrm{d} U_{I}^{P}}{\mathrm{~d} \bar{\sigma}_{\max }}<-\frac{\mu}{1-\mu} \frac{\mathrm{d} U_{U}^{P}}{\mathrm{~d} \bar{\sigma}_{\max }},
$$

a Pareto improvement is always possible through appropriate transfers.

The results are summarized in the following proposition:

Proposition 2 When investment uncertainty eases, the government is able to achieve a Pareto improvement by simultaneously implementing an appropriate lump-sum income transfer from the uninformed to the informed investors. ${ }^{11}$

\footnotetext{
10 One can obtain a closed-form condition by using (B.5) and (B.6) in Appendix B.

11 There are some practical difficulties in implementing such a redistribution. One is that the government has to identify the type of investors. In the present model, the uninformed (informed) investors are those who 


\subsection{Investment subsidies}

One may propose an investment subsidy as an alternative policy instrument for Paretoimproving redistribution because the informed investors demand the risky asset more than the uninformed investors do. However, it is shown not to work.

To demonstrate this, we introduce an investment subsidy financed by a lump-sum taxation. Let us rewrite the individual budget equations as

$$
\bar{m}+\left(p-s^{1}\right) \bar{x}+\tau^{1}=m_{j}+\left(p-s^{1}\right) x_{j}, \quad \tilde{c}_{j}=m_{j}+\left(\tilde{v}+s^{2}\right) x_{j}+\tau^{2},
$$

where $s^{t}$ and $\tau^{t}$ are an investment subsidy rate and a lump-sum subsidy-cum-tax in period $t(=1,2)$, both of which are not discriminated based on the investor's type $j(=I, U)$. In this case, individual utility reduces to

$$
\begin{aligned}
U_{I} & =\bar{m}+\left(p-s^{1}\right) \bar{x}+\tau^{1}+\tau^{2}+\left(\hat{v}-p+s^{1}+s^{2}\right) x_{I}-\frac{\Gamma \hat{\sigma}}{2}\left(x_{I}\right)^{2}, \\
U_{U} & = \begin{cases}\bar{m}+\left(p-s^{1}\right) \bar{x}+\tau^{1}+\tau^{2}+\left(\bar{v}_{\min }-p+s^{1}+s^{2}\right) x_{U}-\frac{\Gamma \bar{\sigma}_{\max }}{2}\left(x_{U}\right)^{2} & \text { if } x_{U} \geq 0 \\
\bar{m}+\left(p-s^{1}\right) \bar{x}+\tau^{1}+\tau^{2}+\left(\bar{v}_{\max }-p+s^{1}+s^{2}\right) x_{U}-\frac{\Gamma \bar{\sigma}_{\max }}{2}\left(x_{U}\right)^{2} & \text { if } x_{U} \leq 0 .\end{cases}
\end{aligned}
$$

See Appendix A for details. For a given asset price $p$, the investment subsidy stimulates investment demand, since $x_{j}$ is increasing with respect to $s^{t}$ :

$$
\begin{aligned}
& x_{I}=\frac{\hat{v}-p+s^{1}+s^{2}}{\Gamma \hat{\sigma}}, \\
& x_{U}= \begin{cases}\frac{\bar{v}_{\min }-p+s^{1}+s^{2}}{\Gamma \bar{\sigma}_{\max }} & \text { if } p<\bar{v}_{\min }+s^{1}+s^{2}, \\
0 & \text { if } \bar{v}_{\min }+s^{1}+s^{2} \leq p \leq \bar{v}_{\max }+s^{1}+s^{2}, \\
\frac{\bar{v}_{\max }-p+s^{1}+s^{2}}{\Gamma \bar{\sigma}_{\max }} & \text { if } \bar{v}_{\max }+s^{1}+s^{2}<p .\end{cases}
\end{aligned}
$$

Applying these demand functions to the equilibrium condition in the risky asset market (8) shows that the expanded asset demand raises the equilibrium asset price, $p^{N}$ and $p^{P}$, proportionately:

$$
p^{N}=\hat{v}-\frac{\Gamma \hat{\sigma} \bar{x}}{1-\mu}+s^{1}+s^{2}, \quad p^{P}=p^{N}+\frac{\mu \Gamma \hat{\sigma}}{1-\mu} x_{U}^{P},
$$

where $x_{U}^{P}$ in the second equation is given by the first equation in (10). Substituting these equilibrium prices into the asset demand functions yields the equilibrium asset holdings of each investor. Consequently, whether the government implements the investment subsidy or not, the resulting demand for the risky asset remains the same as the first and second equations in (9) and (10), and Conditions 1 and 2 are also

Footnote 11 continued

increase (decrease) the risky asset holdings when investment uncertainty is mitigated by policy interventions or information provisions. When such opposite asset positions are observed, a type-dependent transfer is possible. It should be noted that the uninformed (informed) investors are not necessarily poor (rich) people, since the demand functions for the risky asset (5) and (7) are independent of wealth levels. 
unaffected. In sum, the investment subsidy merely pushes up the asset price proportionately.

Let us turn to the welfare analysis. The government finances the investment subsidy by imposing a lump-sum taxation in each period:

$$
s^{1} \bar{x}-\tau^{1}=(1-\mu) s^{1} x_{I}+\mu s^{1} x_{U}, \quad-\tau^{2}=(1-\mu) s^{2} x_{I}+\mu s^{2} x_{U} .
$$

In the equilibrium in which (8) holds, they are

$$
\tau^{1}=0, \quad-\tau^{2}=s^{2} \bar{x}
$$

By applying these relations, the equilibrium demand for the risky asset and the equilibrium asset price to utility, we find that the investment subsidy is neutral to individual as well as social welfare. The result is summarized as follows:

Proposition 3 An investment subsidy financed by a lump-sum taxation pushes up the equilibrium asset price proportionately and thereby is neutral to individual as well as social welfare. Thus, the investment subsidy is not an effective policy instrument to achieve a Pareto improvement.

\section{Government spending on investor education}

In the previous sections, we considered the welfare effect of exogenous changes in the possible mean and variance of investment payoffs perceived by the uninformed investors. However, the policies proposed by Easley and O'Hara (2009), such as investor education, usually require some resources when implemented. Taking into account the physical cost of policy interventions, this section evaluates government spending on education for the uninformed investors.

Given a lump-sum subsidy-cum-taxation $\tau_{j}^{1}$, the investor's budget constraint in each period is

$$
\bar{m}+p \bar{x}+\tau_{j}^{1}=m_{j}+p x_{j}, \quad \tilde{c}_{j}=m_{j}+\tilde{v} x_{j},
$$

which rewrites the investor's utility as

$$
\tilde{U}_{I} \equiv U_{I}+\tau_{I}^{1}, \quad \tilde{U}_{U} \equiv U_{U}+\tau_{U}^{1}
$$

where $U_{I}$ and $U_{U}$ are given in (4) and (6) (see Appendix A for the derivation). It is apparent that the demand functions for the risky asset (5) and (7), the equilibrium values $\left(x_{N}, x_{S}, p\right)$ in (9) and (10), and Conditions 1 and 2 do not alter.

At the beginning of the first period, the uninformed investors perceive the minimum mean payoff to be $\hat{v}_{\text {min }}$. Then, within the first period, the government levies taxes and uses the collected fund to educate the uninformed investors, thereby satisfying the following budget equation:

$$
-\left[(1-\mu) \tau_{I}^{1}+\mu \tau_{U}^{1}\right]=\mu e,
$$


where $e(\geq 0)$ represents per capita education spending by the government. Through public education, the perceived minimum mean payoff is revised to $\bar{v}_{\min }^{\prime}$ and gradually approaches the true mean payoff, $\hat{v}:{ }^{12}$

$$
\bar{v}_{\min }^{\prime}= \begin{cases}\theta e+\bar{v}_{\min } & \text { for } 0 \leq e<\hat{e} \\ \hat{v} & \text { for } e \geq \hat{e}\end{cases}
$$

where input-output coefficient $\theta(>0)$ measures an efficiency of public education. The revised minimum mean payoff, $\bar{v}_{\text {min }}^{\prime}$, cannot exceed the true mean payoff, $\hat{v}$. At the education level $\hat{e}$ defined by

$$
\hat{e} \equiv \frac{\hat{v}-\bar{v}_{\min }}{\theta}(>0),
$$

$\bar{v}_{\min }^{\prime}$ reaches $\hat{v}$. Subject to (18) and (19), the government chooses the education level, $e$, so as to maximize the social welfare,

$$
\begin{aligned}
\tilde{U}_{W} & \equiv(1-\mu) \tilde{U}_{I}+\mu \tilde{U}_{U} \\
& =(1-\mu) U_{I}+\mu U_{U}-\mu e \\
& =U_{W}-\mu e .
\end{aligned}
$$

The physical cost of education spending, $\mu e$, directly reduces the social welfare.

Consider that the economy initially lies in the nonparticipating equilibrium and shifts toward the participating equilibrium by a revision of $\bar{v}_{\min }$. It is optimal to choose $e=0$ as long as the economy remains in the nonparticipating regime, in which public education has no effect on the equilibrium values. Given the (15) in which (20) is applied, we obtain the difference in the social welfare before and after the regime shift:

$$
\tilde{U}_{W}^{P}-\tilde{U}_{W}^{N}=\left\{\begin{array}{l}
f(e)-\mu e \text { for } e_{\min }<e<\hat{e} \\
f(\hat{e})-\mu e \text { for } e \geq \hat{e}
\end{array}\right.
$$

where

$$
\begin{aligned}
f(e) & \equiv \frac{(1-\mu) \mu \theta^{2}}{2 \Gamma\left[(1-\mu) \bar{\sigma}_{\max }+\mu \hat{\sigma}\right]}\left[e-\frac{1}{\theta}\left(\hat{v}-\frac{\Gamma \hat{\sigma} \bar{x}}{1-\mu}-\bar{v}_{\min }\right)\right]^{2}, \\
f(\hat{e}) & =\frac{\mu \Gamma \hat{\sigma}^{2} \bar{x}^{2}}{2(1-\mu)\left[(1-\mu) \bar{\sigma}_{\max }+\mu \hat{\sigma}\right]}
\end{aligned}
$$

The first term on the right-hand side of (21) represents the welfare gain by the regime shift from the nonparticipating equilibrium to the participating equilibrium (see Proposition 1), whereas the second term is the physical cost of education spending. Education

\footnotetext{
12 For the sake of analytical simplicity, we do not consider an effect of public education on the perceived maximum payoff variance. If it is taken into account, the welfare implication basically remains the same.
} 

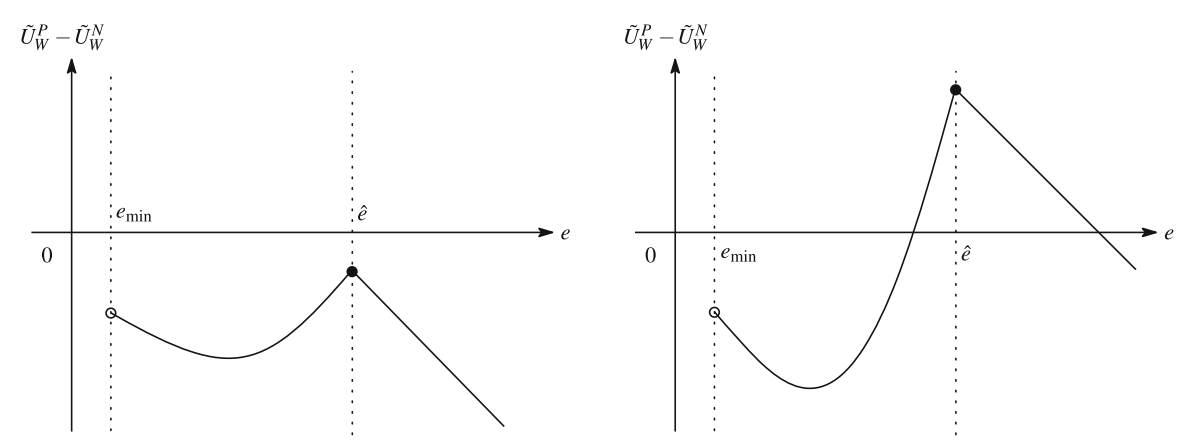

Fig. 1 The shape of $\tilde{U}_{W}^{P}-\tilde{U}_{W}^{N}$. Note: the figure on the left-hand side is the case when $\tilde{U}_{W}^{P}-\tilde{U}_{W}^{N}=$ $f(e)-\mu e \leq 0$ at $e=\hat{e}$, whereas the figure on the right-hand side satisfies $\tilde{U}_{W}^{P}-\tilde{U}_{W}^{N}=f(e)-\mu e>0$ at $e=\hat{e}$

spending enhances the social welfare if (21) has a positive sign. The relation (21) is valid if and only if $\bar{v}_{\min }^{\prime}$ satisfies Condition 2, or equivalently, $e$ lies above the $e_{\min }$ defined by

$$
e_{\min } \equiv \frac{1}{\theta}\left(\hat{v}-\frac{\hat{\sigma} \bar{x}}{1-\mu}-\bar{v}_{\min }\right)(\geq 0)
$$

where $e_{\min }$ is non-negative because $\bar{v}_{\text {min }}$ satisfies Condition 1 . The $e_{\min }$ is the minimum education spending that can shift the economy from the nonparticipating equilibrium to the participating equilibrium.

Figure 1 describes the shape of (21) (see Appendix D for details). Starting from $\tilde{U}_{W}^{P}-\tilde{U}_{W}^{N}=-\mu e<0$ at $e=e_{\min }$, the function (21) is either convex or monotonically decreasing with respect to $e \in\left(e_{\min }, \hat{e}\right)$. This is because public education not only remedies the distortion by uncertainty, as shown in Sect. 3, but also involves the physical cost. For $e \geq \hat{e}$, the function (21) is monotonically decreasing, since public education is no longer effective.

The figure on the left-hand side describes the case in which $\tilde{U}_{W}^{P}-\tilde{U}_{W}^{N}=f(e)-$ $\mu e \leq 0$ at $e=\hat{e}$. Since $\tilde{U}_{W}^{P}$ is less than $\tilde{U}_{W}^{N}$ for $\forall e>e_{\min }$, the optimal policy is not to educate the uninformed investors (i.e., $e=0$ ). Thus, the equilibrium remains in the nonparticipating regime.

The figure on the right-hand side is the case in which $\tilde{U}_{W}^{P}-\tilde{U}_{W}^{N}=f(e)-\mu e>0$ at $e=\hat{e}$. It is optimal to shift the equilibrium to the participating regime by choosing $e=\hat{e}$ so that the uninformed's belief, $\bar{v}_{\text {min }}^{\prime}$, equals the true mean payoff, $\hat{v}$. In other words, the optimal education policy is to assimilate the uninformed investors into the informed investors. This case occurs when the marginal benefit of public education is sufficiently large (see Appendix D). That is,

i) when the efficiency of public education, $\theta$, is sufficiently high;

ii) when the uninformed's asset demand is sufficiently large owing to either high $\bar{v}_{\text {min }}$ or low $\bar{\sigma}_{\text {max }}$; and 
iii) when the informed's asset demand is sufficiently small owing to either low $\hat{v}$ or high $\hat{\sigma}$, implying the low asset price and the large asset demand of the uninformed investors.

In this case, a Pareto improvement is possible by combining the education policy with an appropriate lump-sum transfer, as proven in Proposition 2. Using the government's budget equation (19), the requirement of a Pareto improvement is

$$
\begin{aligned}
& \tilde{U}_{I}^{P}-\tilde{U}_{I}^{N}=\left(U_{I}^{P}-U_{I}^{N}\right)+\tau_{I}^{1} \geq 0 \\
& \tilde{U}_{U}^{P}-\tilde{U}_{U}^{N}=\left(U_{U}^{P}-U_{U}^{N}\right)-\frac{1-\mu}{\mu} \tau_{I}^{1}-\hat{e}>0,
\end{aligned}
$$

or equivalently,

$$
\underbrace{-\left(U_{I}^{P}-U_{I}^{N}\right)}_{(+)} \leq \tau_{I}^{1}<\frac{\mu}{1-\mu}\left[\left(U_{U}^{P}-U_{U}^{N}\right)-\hat{e}\right],
$$

where $U_{I}^{P}-U_{I}^{N}<0$ is from (13). The enlarged social welfare implies

$$
\tilde{U}_{W}^{P}-\tilde{U}_{W}^{N}=(1-\mu)\left(U_{I}^{P}-U_{I}^{N}\right)+\mu\left(U_{U}^{P}-U_{U}^{N}\right)-\mu \hat{e}>0,
$$

or equivalently,

$$
-\left(U_{I}^{P}-U_{I}^{N}\right)<\frac{\mu}{1-\mu}\left[\left(U_{U}^{P}-U_{U}^{N}\right)-\hat{e}\right]
$$

Therefore, there always exists a Pareto-improving transfer from the uninformed to the informed. ${ }^{13}$

Obviously, the same argument is possible for the case when the economy starts from the participating equilibrium. We summarize the result in the following proposition:

\section{Proposition 4}

- In the case with relatively low $\left(\theta, \bar{v}_{\text {min }}, \hat{\sigma}\right)$ and high $\left(\bar{\sigma}_{\text {max }}, \hat{v}\right)$, it is optimal to not educate the uninformed investors.

\footnotetext{
13 A positive $\tau_{I}^{1}$ means that the payment exceeding the education cost is imposed on the uninformed investors, that is, $\tau_{U}^{1} \leq-\hat{e}<0$ from the government's budget equation (19). Even when such a payment is imposed, the uninformed investor has an incentive to be educated while the informed investors does not. Hence, the government can identify the type of investors and implement the type-dependent transfer through the voluntary payment to public education. This type of transfer is also practicable through subsidizing investor education provided by private financial institutions. In general, the less informed is a personal investor and the more informed is an institutional investor, such as investment banks and securities companies. The subsidy to, for example, investment seminars held by financial institutions becomes an indirect transfer from the uninformed to the informed. The government need not identify the type of investors, because the less informed personal investor cannot provide such an opportunity.
} 
- In the case with sufficiently high $\left(\theta, \bar{v}_{\text {min }}, \hat{\sigma}\right)$ and low $\left(\bar{\sigma}_{\text {max }}, \hat{v}\right)$, it is optimal to educate the uninformed investors so that their belief corresponds to the true payoff distribution. A Pareto improvement is possible to be attained by implementing an appropriate income transfer from the uninformed investors to the informed investors.

\section{Conclusion}

Numerous theoretical and empirical studies report considerable effects of uncertainty or ambiguity on financial markets and asset prices. They usually presume that reducing uncertainty over investment payoffs is desirable, since it corrects distorted asset prices. In this study, we examined individuals' welfare to show that, without appropriate income transfers, mitigating the uncertainty does not achieve a Pareto improvement by hurting some investors, while it enhances the social welfare. This result fairly differs from the effects of a shift in the true distribution - for example, a rise in the true mean payoff is definitely beneficial to all individuals even without policy interventions.

We also show that an appropriate lump-sum transfer from the improved investors to the damaged investors is useful to attain a Pareto improvement; on the other hand, an investment subsidy financed by a lump-sum taxation is not an effective instrument for Pareto-improving redistribution. Furthermore, we analyze the welfare effect of investor education, which is optimally chosen by the government and endogenously determines the degree of ambiguity perceived by the uninformed investors. When combined with an appropriate transfer, public education may achieve a Pareto improvement.

While the simple model used in this study achieves a main part of our aim, there are some directions to be extended for further research. First, we can determine implications for economic growth and poverty traps by developing a dynamic model with capital accumulation, such as that done by Fukuda (2008). Second, it is important to analyze an incentive of privately incurring education costs and a learning process by the uninformed investors. ${ }^{14}$ Third, to assess quantitative effects, we have to build a larger scale model and estimate structural parameters that include the probability distribution perceived by the uninformed investors.

Funding This research is financially supported by JSPS KAKENHI Grant Numbers 19K01767 and 20K01669 for Ogawa and 19K23212 and 20K01669 for Sakamoto.

\section{Declaration}

Conflict of interest The authors declare that they have no conflict of interest.

Open Access This article is licensed under a Creative Commons Attribution 4.0 International License, which permits use, sharing, adaptation, distribution and reproduction in any medium or format, as long as you give appropriate credit to the original author(s) and the source, provide a link to the Creative Commons licence, and indicate if changes were made. The images or other third party material in this article are included in the article's Creative Commons licence, unless indicated otherwise in a credit line to the material. If

\footnotetext{
14 Mele and Sangiorgi (2015) consider an incentive to reduce investment uncertainty by costly acquisition of information on the payoff distribution and find that the price swings occur.
} 
material is not included in the article's Creative Commons licence and your intended use is not permitted by statutory regulation or exceeds the permitted use, you will need to obtain permission directly from the copyright holder. To view a copy of this licence, visit http://creativecommons.org/licenses/by/4.0/.

\section{Appendix A: utility maximization problems}

This appendix proves that the utility maximization problems are represented by the mean-variance approach, (4) and (6). Consider the following constant absolute risk aversion utility function, $u(\cdot)$ :

$$
\mathrm{E}_{j}\left[u\left(\tilde{c}_{j}\right)\right]=\mathrm{E}_{j}\left[-\exp \left(-\Gamma \tilde{c}_{j}\right)\right] \quad \text { for } j=I, U
$$

Note that, in the presence of uncertainty, the expectation operator takes a different form between the informed and the uninformed. Since the stochastic process of $\tilde{c}_{j}$ follows the normal distribution, the utility reduces to

$$
\mathrm{E}_{j}\left[u\left(\tilde{c}_{j}\right)\right]=-\exp \left(-\Gamma U_{j}\right), \quad \text { where } \quad U_{j} \equiv \mathrm{E}_{j}\left[\tilde{c}_{j}\right]-\frac{\Gamma}{2} \operatorname{Var}_{j}\left[\tilde{c}_{j}\right]
$$

The expected utility thus increases with $U_{j}$.

From (3), the subjective expected mean and variance of $\tilde{c}_{j}$ are respectively given by

$$
\begin{aligned}
\mathrm{E}_{j}\left[\tilde{c}_{j}\right] & =m_{j}+\mathrm{E}_{j}[\tilde{v}] x_{j} \\
& =(\bar{m}+p \bar{x})+\left(\mathrm{E}_{j}[\tilde{v}]-p\right) x_{j}, \quad \operatorname{Var}_{j}\left[\tilde{c}_{j}\right]=\operatorname{Var}_{j}[\tilde{v}]\left(x_{j}\right)^{2},
\end{aligned}
$$

where the second equality in the first equation comes from (2).

The informed investor knows the true probability distribution of investment payoffs, so that we have

$$
\mathrm{E}_{I}[\tilde{v}]=\hat{v}, \quad \operatorname{Var}_{I}[\tilde{v}]=\hat{\sigma}
$$

The uninformed does not know the true distribution and considers the worst case among the possible set of mean payoff and variance to avoid ambiguity (see Gilboa and Schmeidler 1989):

$$
\mathrm{E}_{U}[\tilde{v}]=\left\{\begin{array}{ll}
\bar{v}_{\text {min }} & \text { if } x_{U} \geq 0, \\
\bar{v}_{\text {max }} & \text { if } x_{U} \leq 0,
\end{array} \quad \operatorname{Var}_{N}[\tilde{v}]=\bar{\sigma}_{\text {max }}\right.
$$

The first equation implies that the worst case of possible mean payoff depends on a long and short position. Substituting (A.2) into the $U_{j}$ in (A.1) and applying either (A.3) or (A.4) to the result yields (4) and (6) in the text. 
In Sects. 4 and 5, we introduce a lump-sum subsidy-cum-taxation $\tau_{j}^{t}$ and an investment subsidy rate $s_{j}^{t}(t=1,2$ and $j=I, U)$. In this case, (A.2) is replaced by

$$
\begin{aligned}
\mathrm{E}_{j}\left[\tilde{c}_{j}\right] & =\bar{m}+\tau_{j}^{1}+\tau_{j}^{2}+\left(p-s^{1}\right) \bar{x}+\left(\mathrm{E}_{j}[\tilde{v}]-p+s^{1}+s^{2}\right) x_{j}, \\
\operatorname{Var}_{j}\left[\tilde{c}_{j}\right] & =\operatorname{Var}_{j}[\tilde{v}]\left(x_{j}\right)^{2} .
\end{aligned}
$$

By substituting these equations into the $U_{j}$ in (A.1) and using the government's budget equation, we can redefine the utility as presented in Sects. 4 and 5. Differentiating the utility with respect to $x_{j}$ implies that: (i) the lump-sum transfer $\tau_{j}^{t}$ does not influence the demand function for $x_{j}$, but does influence the level of individual welfare (see Sects. 4.1 and 5); and (ii) the demand function for $x_{j}$ is affected by the investment subsidy $s_{j}^{t}$ (see Sect. 4.2).

\section{Appendix B: Proof of Proposition 1}

This appendix provides a mathematical proof of Proposition 1. It is obvious that the sign of (11) is ensured by the third equation in (10). If needed, we can present a closedform solution by substituting the third and first equations in (10) to eliminate $p^{P}$ and $x_{U}^{P}$ from (11):

$$
U_{U}^{P}-U_{U}^{N}=\frac{x_{U}^{P}}{\mu}\left\{\frac{\mu^{2} \Gamma \hat{\sigma} \bar{x}}{1-\mu}+\frac{\mu(1-\mu) \bar{\sigma}_{\max }}{2\left[(1-\mu) \bar{\sigma}_{\max }+\mu \hat{\sigma}\right]}\left[\bar{v}_{\min }^{\prime}-\left(\hat{v}-\frac{\Gamma \hat{\sigma} \bar{x}}{1-\mu}\right)\right]\right\}>0,
$$

which is positive under Condition 2 in which $\bar{v}_{\min }$ is replaced by $\bar{v}_{\min }^{\prime}$.

To obtain (13), we eliminate $p^{P}$ and $x_{I}^{P}$ from (12) by using the third and second equations in (10):

$$
U_{I}^{P}-U_{I}^{N}=\frac{\mu \Gamma \hat{\sigma} x_{U}^{P}}{1-\mu}\left[\bar{x}-x_{I}^{N}+\frac{\mu}{2(1-\mu)} x_{U}^{P}\right]
$$

Eliminating $x_{I}^{N}$ and $x_{U}^{P}$ from the bracket in the second equality by substituting the second equation in (9) and the first equation in (10), we obtain (13) in the text, or equivalently,

$$
U_{I}^{P}-U_{I}^{N}=-\frac{x_{U}^{P}}{1-\mu}\left\{\frac{\mu^{2} \Gamma \hat{\sigma} \bar{x}}{1-\mu}-\frac{\mu^{2} \hat{\sigma}}{2\left[(1-\mu) \bar{\sigma}_{\max }+\mu \hat{\sigma}\right]}\left[\bar{v}_{\min }^{\prime}-\left(\hat{v}-\frac{\Gamma \hat{\sigma} \bar{x}}{1-\mu}\right)\right]\right\} .
$$

We now derive (15) in the text. Equation (14) implies

$$
U_{W}^{P}-U_{W}^{N}=(1-\mu)\left(U_{I}^{P}-U_{I}^{N}\right)+\mu\left(U_{U}^{P}-U_{U}^{N}\right)
$$

Applying (B.5) and (B.6) to this equation and using the first equation in (10) to eliminate $x_{U}^{P}$ from the result yields (15) in the text. 
Let us turn to comparative statics within the participating equilibrium. Remember that $x_{I}^{N}$ and $p^{N}$ in the second and third equations in (10) are independent of $\bar{v}_{\text {min }}$ and $\bar{\sigma}_{\max }$. The total differentiation of (10) and (14) with respect to $\bar{v}_{\text {min }}$ generates

$$
\begin{aligned}
\frac{\mathrm{d} x_{U}^{P}}{\mathrm{~d} \bar{v}_{\text {min }}} & =\frac{1-\mu}{\Gamma\left[(1-\mu) \bar{\sigma}_{\text {max }}+\mu \hat{\sigma}\right]}>0, \\
\frac{\mathrm{d} x_{I}^{P}}{\mathrm{~d} \bar{v}_{\text {min }}} & =-\frac{\mu}{1-\mu} \frac{\mathrm{d} x_{U}^{P}}{\mathrm{~d} \bar{v}_{\text {min }}}<0, \\
\frac{\mathrm{d} p^{P}}{\mathrm{~d} \bar{v}_{\text {min }}} & =\frac{\mu \Gamma \hat{\sigma}}{1-\mu} \frac{\mathrm{d} x_{U}^{P}}{\mathrm{~d} \bar{v}_{\text {min }}}>0, \\
\frac{\mathrm{d} U_{U}^{P}}{\mathrm{~d} \bar{v}_{\min }} & =\bar{x} \frac{\mathrm{d} p^{P}}{\mathrm{~d} \bar{v}_{\text {min }}}+\Gamma \bar{\sigma}_{\max } x_{U}^{P} \frac{\mathrm{d} x_{U}^{P}}{\mathrm{~d} \bar{v}_{\min }}>0, \\
\frac{\mathrm{d} U_{I}^{P}}{\mathrm{~d} \bar{v}_{\min }} & =-\frac{\mu^{2} \hat{\sigma}\left[\left(\hat{v}-\bar{v}_{\min }\right)+\Gamma\left(\bar{\sigma}_{\max }-\hat{\sigma}\right) \bar{x}\right]}{(1-\mu)\left[(1-\mu) \bar{\sigma}_{\max }+\mu \hat{\sigma}\right]} \frac{\mathrm{d} x_{U}^{P}}{\mathrm{~d} \bar{v}_{\min }}<0, \\
\frac{\mathrm{d} U_{W}^{P}}{\mathrm{~d} \bar{v}_{\min }} & =\mu x_{U}^{P}>0,
\end{aligned}
$$

where the sign of the fifth equation is determined by (1). Similarly, we totally differentiate (10) and (14) with respect to $\bar{\sigma}_{\max }$ to have

$$
\begin{aligned}
\frac{\mathrm{d} x_{U}^{P}}{\mathrm{~d} \bar{\sigma}_{\max }} & =-\frac{(1-\mu)^{2}}{\Gamma\left[(1-\mu) \bar{\sigma}_{\max }+\mu \hat{\sigma}\right]^{2}}\left[\bar{v}_{\min }-\left(\hat{v}-\frac{\Gamma \hat{\sigma} \bar{x}}{1-\mu}\right)\right]<0, \\
\frac{\mathrm{d} x_{I}^{P}}{\mathrm{~d} \bar{\sigma}_{\max }} & =-\frac{\mu}{1-\mu} \frac{\mathrm{d} x_{U}^{P}}{\mathrm{~d} \bar{\sigma}_{\max }}>0, \\
\frac{\mathrm{d} p^{P}}{\mathrm{~d} \bar{\sigma}_{\max }} & =\frac{\mu \Gamma \hat{\sigma}}{1-\mu} \frac{\mathrm{d} x_{U}^{P}}{\mathrm{~d} \bar{\sigma}_{\max }}<0, \\
\frac{\mathrm{d} U_{U}^{P}}{\mathrm{~d} \bar{\sigma}_{\max }} & =\frac{A}{2\left[(1-\mu) \bar{\sigma}_{\max }+\mu \hat{\sigma}\right]} \frac{\mathrm{d} x_{U}^{P}}{\mathrm{~d} \bar{\sigma}_{\max }}<0, \\
\frac{\mathrm{d} U_{I}^{P}}{\mathrm{~d} \bar{\sigma}_{\max }} & =-\frac{\mu^{2} \hat{\sigma}\left[\left(\hat{v}-\bar{v}_{\min }\right)+\Gamma\left(\bar{\sigma}_{\max }-\hat{\sigma}\right) \bar{x}\right]}{(1-\mu)\left[(1-\mu) \bar{\sigma}_{\max }+\mu \hat{\sigma}\right]} \frac{\mathrm{d} x_{U}^{P}}{\mathrm{~d} \bar{\sigma}_{\max }}>0, \\
\frac{\mathrm{d} U_{W}^{P}}{\mathrm{~d} \bar{\sigma}_{\max }} & =-\frac{\mu \Gamma\left(x_{U}^{P}\right)^{2}}{2}<0,
\end{aligned}
$$

where

$$
\begin{aligned}
A \equiv & {\left[(1-\mu) \bar{\sigma}_{\max }+\mu \hat{\sigma}\right]\left[\bar{v}_{\min }-\left(\hat{v}-\frac{\Gamma \hat{\sigma} \bar{x}}{1-\mu}\right)\right] } \\
& +2 \mu \hat{\sigma}\left[\left(\hat{v}-\bar{v}_{\min }\right)+\Gamma\left(\bar{\sigma}_{\max }-\hat{\sigma}\right) \bar{x}\right]>0 .
\end{aligned}
$$

Under Condition 2, the first equation is to be negative. Condition 2 and (1) ensure a positive sign of $A$ and thus determine the sign of the fourth equation. The fifth equation has a positive value owing to (1). 


\section{Appendix C: Implications of a shift in the true payoff distribution}

This appendix derives welfare effects of a shift in the true payoff distribution, represented by a rise in the true mean payoff from $\hat{v}$ to $\hat{v}^{\prime}(>\hat{v})$ and a fall in the true payoff variance from $\hat{\sigma}$ to $\hat{\sigma}^{\prime}(<\hat{\sigma})$.

We first conduct a comparative static analysis within the nonparticipating equilibrium. From (9), we have

$$
\frac{\mathrm{d} x_{U}^{N}}{\mathrm{~d} \hat{v}}=\frac{\mathrm{d} x_{I}^{N}}{\mathrm{~d} \hat{v}}=0, \quad \frac{\mathrm{d} p^{N}}{\mathrm{~d} \hat{v}}=1>0, \quad \frac{\mathrm{d} U_{U}^{N}}{\mathrm{~d} \hat{v}}=\frac{\mathrm{d} U_{I}^{N}}{\mathrm{~d} \hat{v}}=\bar{x}>0 .
$$

A rise in $\hat{v}$ stimulates the informed's asset demand and raises the asset price. This is desirable for all people by increasing the value of the initial endowment. It is noteworthy to mention a difference between effects of changes in $\hat{v}$ and $\bar{v}_{\min }$ - the expectation formed by the uninformed investor, $\bar{v}_{\min }$, has no effect on the nonparticipating equilibrium. $\hat{\sigma}$ also influences the nonparticipating equilibrium:

$$
\begin{aligned}
& \frac{\mathrm{d} x_{U}^{N}}{\mathrm{~d} \hat{\sigma}}=\frac{\mathrm{d} x_{I}^{N}}{\mathrm{~d} \hat{\sigma}}=0, \quad \frac{\mathrm{d} p^{N}}{\mathrm{~d} \hat{\sigma}}=-\frac{\Gamma \bar{x}}{1-\mu}<0, \\
& \frac{\mathrm{d} U_{U}^{N}}{\mathrm{~d} \hat{\sigma}}=-\frac{\Gamma \bar{x}^{2}}{1-\mu}<0, \quad \frac{\mathrm{d} U_{I}^{N}}{\mathrm{~d} \hat{\sigma}}=-\frac{(1-2 \mu) \Gamma \bar{x}^{2}}{2(1-\mu)^{2}} \begin{cases}<0 & \text { if } 0<\mu<\frac{1}{2}, \\
=0 & \text { if } \mu=\frac{1}{2}, \\
>0 & \text { if } \frac{1}{2}<\mu<1 .\end{cases}
\end{aligned}
$$

A fall in $\hat{\sigma}$ not only raises the risky asset price, $p$, but also reduces the excess return of holding the risky asset, as illustrated in the third equation in (9):

$$
\hat{v}-p^{N}=\frac{\Gamma \hat{\sigma} \bar{x}}{1-\mu}
$$

Thus, the welfare effect on the informed investor is generically ambiguous. In the case in which $0<\mu<\frac{1}{2}$, a Pareto improvement is attained even with no policy intervention - for the informed investor, the beneficial effect from the increased value of the initial endowment outweighs the harmful effect from the reduced excess return.

From Conditions 1 and 2, both a rise in $\hat{v}$ and a fall in $\hat{\sigma}$ shift the economy from the participating equilibrium (Case 2 ) to the nonparticipating equilibrium (Case 1 ) by raising the risky asset price. Evaluated around the threshold satisfying $\hat{v}-\frac{\Gamma \hat{\sigma} \bar{x}}{1-\mu} \approx \bar{v}_{\min }$, the local effects are derived as follows: from (9) and (10),

$$
\frac{x_{U}^{N}-x_{U}^{P}}{\hat{v}^{\prime}-\hat{v}}=\frac{x_{I}^{N}-x_{I}^{P}}{\hat{v}^{\prime}-\hat{v}}=0, \quad \frac{p^{N}-p^{P}}{\hat{v}^{\prime}-\hat{v}}=1>0, \quad \frac{U_{I}^{N}-U_{I}^{P}}{\hat{v}^{\prime}-\hat{v}}=\frac{U_{U}^{N}-U_{U}^{P}}{\hat{v}^{\prime}-\hat{v}}=\bar{x}>0,
$$

and

$$
\frac{x_{U}^{N}-x_{U}^{P}}{\hat{\sigma}^{\prime}-\hat{\sigma}}=\frac{x_{I}^{N}-x_{I}^{P}}{\hat{\sigma}^{\prime}-\hat{\sigma}}=0, \quad \frac{p^{N}-p^{P}}{\hat{\sigma}^{\prime}-\hat{\sigma}}=-\frac{\Gamma \bar{x}}{1-\mu}<0,
$$




$$
\frac{U_{U}^{N}-U_{U}^{P}}{\hat{\sigma}^{\prime}-\hat{\sigma}}=-\frac{\Gamma \bar{x}^{2}}{1-\mu}<0, \quad \frac{U_{I}^{N}-U_{I}^{P}}{\hat{\sigma}^{\prime}-\hat{\sigma}}=-\frac{(1-2 \mu) \Gamma \bar{x}^{2}}{2(1-\mu)^{2}} \begin{cases}<0 & \text { if } 0<\mu<\frac{1}{2} \\ =0 & \text { if } \mu=\frac{1}{2} \\ >0 & \text { if } \frac{1}{2}<\mu<1\end{cases}
$$

The welfare implications are exactly the same as those within the nonparticipating equilibrium.

We turn to the analysis within the participating equilibrium. The total differentiation of (10) with respect to $\hat{v}$ yields

$$
\begin{aligned}
\frac{\mathrm{d} x_{I}^{P}}{\mathrm{~d} \hat{v}} & =\frac{\mu}{\Gamma\left[(1-\mu) \bar{\sigma}_{\max }+\mu \hat{\sigma}\right]}>0, \\
\frac{\mathrm{d} x_{U}^{P}}{\mathrm{~d} \hat{v}} & =-\frac{1-\mu}{\mu} \frac{\mathrm{d} x_{I}^{P}}{\mathrm{~d} \hat{v}}<0, \\
\frac{\mathrm{d} p^{P}}{\mathrm{~d} \hat{v}} & =\frac{(1-\mu) \Gamma \bar{\sigma}_{\max }}{\mu} \frac{\mathrm{d} x_{I}^{P}}{\mathrm{~d} \hat{v}}>0, \\
\frac{\mathrm{d} U_{I}^{P}}{\mathrm{~d} \hat{v}} & =\bar{x} \frac{\mathrm{d} p^{P}}{\mathrm{~d} \hat{v}}+\Gamma \hat{\sigma} x_{I}^{P} \frac{\mathrm{d} x_{I}^{P}}{\mathrm{~d} \hat{v}}>0, \\
\frac{\mathrm{d} U_{I}^{P}}{\mathrm{~d} \hat{v}} & =\bar{x} \frac{\mathrm{d} p^{P}}{\mathrm{~d} \hat{v}}+\Gamma \bar{\sigma}_{\max } x_{U}^{P} \frac{\mathrm{d} x_{U}^{P}}{\mathrm{~d} \hat{v}} \\
& =\frac{(1-\mu)^{2} \bar{\sigma}_{\max }\left[\left(\hat{v}-\bar{v}_{\min }\right)+\Gamma\left(\bar{\sigma}_{\max }-\hat{\sigma}\right) \bar{x}\right]}{\mu\left[(1-\mu) \bar{\sigma}_{\max }+\mu \hat{\sigma}\right]} \frac{\mathrm{d} x_{I}^{P}}{\mathrm{~d} \hat{v}}>0,
\end{aligned}
$$

where the sign of the last equation is ensured by (1). Thus, a rise in $\hat{v}$ always achieves a Pareto improvement within the participating equilibrium, implying that, for the uninformed investor, the beneficial effect from the increased value of the initial endowment definitely outweighs the harmful effect from the reduced risky asset holdings. On the other hand, we have

$$
\begin{aligned}
\frac{\mathrm{d} x_{I}^{P}}{\mathrm{~d} \hat{\sigma}} & =-\frac{\mu^{2}}{\Gamma\left[(1-\mu) \bar{\sigma}_{\max }+\mu \hat{\sigma}\right]^{2}}\left[\left(\hat{v}-\bar{v}_{\min }\right)+\frac{\Gamma \bar{\sigma}_{\max } \bar{x}}{\mu}\right]<0, \\
\frac{\mathrm{d} x_{U}^{P}}{\mathrm{~d} \hat{\sigma}} & =-\frac{1-\mu}{\mu} \frac{\mathrm{d} x_{I}^{P}}{\mathrm{~d} \hat{\sigma}}>0, \\
\frac{\mathrm{d} p^{P}}{\mathrm{~d} \hat{\sigma}} & =\frac{(1-\mu) \Gamma \bar{\sigma}_{\max }}{\mu} \frac{\mathrm{d} x_{I}^{P}}{\mathrm{~d} \hat{\sigma}}<0, \\
\frac{\mathrm{d} U_{I}^{P}}{\mathrm{~d} \hat{\sigma}} & =\frac{B}{2\left[(1-\mu) \bar{\sigma}_{\max }+\mu \hat{\sigma}\right]} \frac{\mathrm{d} x_{I}^{P}}{\mathrm{~d} \hat{\sigma}} \lesseqgtr 0, \\
\frac{\mathrm{d} U_{U}^{P}}{\mathrm{~d} \hat{\sigma}} & =\frac{\left.(1-\mu)^{2} \bar{\sigma}_{\max }\left[\left(\hat{v}-\bar{v}_{\min }\right)+\Gamma\left(\bar{\sigma}_{\max }-\hat{\sigma}\right) \bar{x}\right]\right\}}{\mu\left[(1-\mu) \bar{\sigma}_{\max }+\mu \hat{\sigma}\right]} \frac{\mathrm{d} x_{I}^{P}}{\mathrm{~d} \hat{\sigma}}<0,
\end{aligned}
$$

where 


$$
\begin{aligned}
B \equiv & {\left[(1-\mu) \bar{\sigma}_{\max }+\mu \hat{\sigma}\right]\left[\left(\hat{v}-\bar{v}_{\min }\right)+\frac{\Gamma \bar{\sigma}_{\max } \bar{x}}{\mu}\right] } \\
& -2(1-\mu) \bar{\sigma}_{\max }\left[\left(\hat{v}-\bar{v}_{\min }\right)+\Gamma\left(\bar{\sigma}_{\max }-\hat{\sigma}\right) \bar{x}\right] \gtreqless 0, \\
\lim _{\mu \rightarrow 0} \frac{\mathrm{d} U_{I}^{P}}{\mathrm{~d} \hat{\sigma}}= & -\frac{\Gamma \bar{x}^{2}}{2}<0 .
\end{aligned}
$$

As in the previous results obtained within the nonparticipating equilibrium, the informed's welfare may or may not improve owing to the decreased excess return of holding the risky asset. However, we can show that a rise in $\hat{\sigma}$ is Pareto-improving at least for a small $\mu$.

We conclude that, regardless of whether the economy lies in the nonparticipating equilibrium or in the participating equilibrium, (i) a rise in $\hat{v}$ achieves a Pareto improvement even without policy interventions and (ii) a fall in $\hat{\sigma}$ is Pareto-improving at least for a small $\mu$.

\section{Appendix D: The Property of (21)}

This appendix presents the property of (21). At $e=e_{\min }$, the asset demand function of each investor and the asset price are the same between the two types of equilibria, except for the education cost incurred in the participating equilibrium. Thus, (21) has a negative value at $e=e_{\min }$ :

$$
\left.\left(\tilde{U}_{W}^{P}-\tilde{U}_{W}^{N}\right)\right|_{e=e_{\min }}=-\mu e_{\min }<0
$$

Differentiating (21) with respect to $e$ yields

$$
\begin{aligned}
& \frac{\mathrm{d}\left(\tilde{U}_{W}^{P}-\tilde{U}_{W}^{N}\right)}{\mathrm{d} e}= \begin{cases}\frac{(1-\mu) \mu \theta^{2}}{\Gamma\left[(1-\mu) \bar{\sigma}_{\max }+\mu \hat{\sigma}\right]}\left[e-\frac{1}{\theta}\left(\hat{v}-\frac{\Gamma \hat{\sigma} \bar{x}}{1-\mu}-\bar{v}_{\min }\right)\right]-\mu & \text { for } e \in\left(e_{\min }, \hat{e}\right), \\
-\mu<0 & \text { for } e \geq \hat{e},\end{cases} \\
& \frac{\mathrm{d}^{2}\left(\tilde{U}_{W}^{P}-\tilde{U}_{W}^{N}\right)}{\mathrm{d} e^{2}}= \begin{cases}\frac{(1-\mu) \mu \theta^{2}}{\Gamma\left[(1-\mu) \bar{\sigma}_{\max }+\mu \hat{\sigma}\right]}>0 & \text { for } e \in\left(e_{\min }, \hat{e}\right), \\
0 & \text { for } e \geq \hat{e} .\end{cases}
\end{aligned}
$$

Let us now define the $e$ that gives $\frac{\mathrm{d}}{\mathrm{d} e}\left(\tilde{U}_{W}^{P}-\tilde{U}_{W}^{N}\right)=0$ :

$$
e^{*} \equiv \frac{1}{\theta}\left\{\hat{v}-\frac{\Gamma \hat{\sigma} \bar{x}}{1-\mu}-\bar{v}_{\min }+\frac{\Gamma\left[(1-\mu) \bar{\sigma}_{\max }+\mu \hat{\sigma}\right]}{(1-\mu) \theta}\right\}\left(>e_{\min } \geq 0\right) .
$$

Since $e^{*}$ is larger than $e_{\min }$, there are two cases regarding the functional form. First, if $e^{*} \geq \hat{e}$, or equivalently, if $(1-\mu) \bar{\sigma}_{\max }+\mu \hat{\sigma} \geq \theta \hat{\sigma} \bar{x}$, then (21) is downward sloping for $\forall e\left(>e_{\min }\right)$ and always has a negative value. This case is not depicted in Fig. 1, but we can categorize it into the figure on the left because the optimal level of public education is zero. 
Second, if $e^{*}<\hat{e}$, or equivalently, if $(1-\mu) \bar{\sigma}_{\max }+\mu \hat{\sigma}<\theta \hat{\sigma} \bar{x}$, then (21) is convex for $e \in\left(e_{\min }, \hat{e}\right)$ and is monotonically decreasing for $e \geq \hat{e}$. In Fig. 1, the figure on the left is the case in which $\tilde{U}_{W}^{P}-\tilde{U}_{W}^{N}$ always has a non-positive value, satisfying

$$
\left.\left(\tilde{U}_{W}^{P}-\tilde{U}_{W}^{N}\right)\right|_{e=\hat{e}}=\frac{\mu \Gamma \hat{\sigma}^{2} \bar{x}^{2}}{2(1-\mu)\left[(1-\mu) \bar{\sigma}_{\max }+\mu \hat{\sigma}\right]}-\frac{\mu\left(\hat{v}-\bar{v}_{\min }\right)}{\theta} \leq 0 .
$$

In contrast, the figure on the right satisfies

$$
\left.\left(\tilde{U}_{W}^{P}-\tilde{U}_{W}^{N}\right)\right|_{e=\hat{e}}=\frac{\mu \Gamma \hat{\sigma}^{2} \bar{x}^{2}}{2(1-\mu)\left[(1-\mu) \bar{\sigma}_{\max }+\mu \hat{\sigma}\right]}-\frac{\mu\left(\hat{v}-\bar{v}_{\min }\right)}{\theta}>0,
$$

and thus $\tilde{U}_{W}^{P}-\tilde{U}_{W}^{N}$ has a positive and maximum value at $e=\hat{e}$. This case occurs with sufficiently high $\left(\theta, \bar{v}_{\min }, \hat{\sigma}\right)$ and low $\left(\bar{\sigma}_{\max }, \hat{v}\right)$ because:

$$
\begin{aligned}
& \left.\frac{\mathrm{d}\left(\tilde{U}_{W}^{P}-\tilde{U}_{W}^{N}\right)}{\mathrm{d} \theta}\right|_{e=\hat{e}}=\frac{\mu\left(\hat{v}-\bar{v}_{\min }\right)}{\theta^{2}}>0, \\
& \left.\frac{\mathrm{d}\left(\tilde{U}_{W}^{P}-\tilde{U}_{W}^{N}\right)}{\mathrm{d} \bar{v}_{\min }}\right|_{e=\hat{e}}=\frac{\mu}{\theta}>0, \\
& \left.\frac{\mathrm{d}\left(\tilde{U}_{W}^{P}-\tilde{U}_{W}^{N}\right)}{\mathrm{d} \bar{\sigma}_{\max }}\right|_{e=\hat{e}}=-\frac{\mu \Gamma \hat{\sigma}^{2} \bar{x}^{2}}{2\left[(1-\mu) \bar{\sigma}_{\max }+\mu \hat{\sigma}\right]^{2}}<0, \\
& \left.\frac{\mathrm{d}\left(\tilde{U}_{W}^{P}-\tilde{U}_{W}^{N}\right)}{\mathrm{d} \hat{v}}\right|_{e=\hat{e}}=-\frac{\mu}{\theta}<0, \\
& \left.\frac{\mathrm{d}\left(\tilde{U}_{W}^{P}-\tilde{U}_{W}^{N}\right)}{\mathrm{d} \hat{\sigma}}\right|_{e=\hat{e}}=\frac{\mu \Gamma \hat{\sigma} \bar{x}^{2}\left[2(1-\mu) \bar{\sigma}_{\max }+\mu \hat{\sigma}\right]}{2(1-\mu)\left[(1-\mu) \bar{\sigma}_{\max }+\mu \hat{\sigma}\right]^{2}}>0 .
\end{aligned}
$$

\section{References}

Alonso, I., Prado, M.: Ambiguity aversion, asset prices, and the welfare costs of aggregate fluctuations. J. Econ. Dyn. Control 51, 78-92 (2015)

Anderson, E.W., Ghysels, E., Juergens, J.L.: The impact of risk and uncertainty on expected returns. J. Financ. Econ. 94, 233-263 (2009)

Antoniou, C., Harris, R., Zhang, R.: Ambiguity aversion and stock market participation: an empirical analysis. J. Bank. Financ. 58, 57-70 (2015)

Bidder, R.M., Smith, M.E.: Robust animal spirits. J. Monet. Econ. 59, 738-750 (2012)

Cagetti, M., Hansen, L.P., Sargent, T., Williams, N.: Robustness and pricing with uncertain growth. Rev. Financ. Stud. 15, 363-404 (2002)

Cao, H.H., Wang, T., Zhang, H.H.: Model uncertainty, limited market participation and asset prices. Rev. Financ. Stud. 18, 1219-1251 (2005) 
Chen, Z., Epstein, L.: Ambiguity, risk, and asset payoffs in continuous time. Econometrica 70, 1403-1443 (2002)

Chen, H., Ju, N., Miao, J.: Dynamic asset allocation with ambiguous return predictability. Rev. Econ. Dyn. 17, 799-823 (2014)

Dow, J., da Costa Werlang, S.R.: Uncertainty aversion, risk aversion, and the optimal choice of portfolio. Econometrica 60, 197-204 (1992)

Easley, D., OHara, M.: Ambiguity and nonparticipation: The role of regulation. Rev. Financ. Stud. 22, 1817-1843 (2009)

Easley, D., OHara, M., Yang, L.: Opaque trading, disclosure, and asset prices: Implication for hedge fund regulation. Rev. Financ. Stud. 27, 1190-1237 (2014)

Epstein, L.G., Miao, J.: A two-person dynamic equilibrium under ambiguity. J. Econ. Dyn. Control 27, $1253-1288(2003)$

Epstein, L.G., Wang, T.: Intertemporal asset pricing under Knightian uncertainty. Econometrica 62, 283-322 (1994)

Epstein, L.G., Wang, T.: Uncertainty, risk-neutral measures and security price booms and crashes. J. Econ. Theor. 67, 40-82 (1995)

Fukuda, S.: Knightian uncertainty and poverty trap in a model of economic growth. Rev. Econ. Dyn. 11, 652-663 (2008)

Gilboa, I., Schmeidler, D.: Maxmin expected utility with non-unique prior. J. Math. Econ. 18, 141-153 (1989)

Guidolin, M., Rinaldi, F.: Ambiguity in asset pricing and portfolio choice: a review of the literature. Theor. Decis. 74, 183-217 (2013)

Huang, H.H., Zhang, S., Zhu, W.: Limited participation under ambiguity of correlation. J. Finan. Mark 32, 97-143 (2017)

Ilut, C.L., Schneider, M.: Ambiguous business cycles. Am. Econ. Rev. 104, 2368-2399 (2014)

Keynes, J.M.: The General Theory of Employment. Interest and Money. MacMillan, London (1937)

Knight, F.H.: Risk, Uncertainty and Profit. Houghton Mifflin, Boston (1921)

Mele, A., Sangiorgi, F.: Uncertainty, information acquisition, and price swings in asset markets. Rev. Financ. Stud. 82, 1533-1567 (2015)

Schmeidler, D.: Subjective probability and expected utility without additivity. Econometrica 57, 571-587 (1989)

Publisher's Note Springer Nature remains neutral with regard to jurisdictional claims in published maps and institutional affiliations. 